\title{
PARALLEL SOLUTION-ADAPTIVE SCHEME FOR MULTI-PHASE CORE FLOWS IN ROCKET MOTORS
}

\author{
J. S. Sachdev*, C. P. T. Groth†, and J. J. Gottlieb \\ University of Toronto Institute for Aerospace Studies \\ Toronto, Ontario, M3H 5T6, Canada
}

\begin{abstract}
The development of a parallel adaptive mesh refinement (AMR) scheme is described for solving the governing equations for multi-phase (gas-particle) core flows in solid propellant rocket motors (SRM). An Eulerian formulation is used for both the gas and particle phases, which leads to a degenerate hyperbolic system of partial differential equations. The cause and effect of the degeneracy is examined. A cell-centered upwind finite-volume discretization and the use of limited solution reconstruction, Riemann solver based flux functions for the gas and particle phases, and explicit multi-stage timestepping allows for high solution accuracy and computational robustness. A Riemann problem is formulated for prescribing boundary data at the burning surface of the propellant grain and an iterative solver is proposed for its solution. Efficient and scalable parallel implementations are achieved with domain decomposition on distributed memory multi-processor architectures. High-scalability of the model has been achieved on a Beowulf-class cluster consisting of $\mathbf{1 0 4}$ processors. Numerical results are described to demonstrate the capabilities of the approach for predicting SRM core flows.
\end{abstract}

\section{Introduction}

$\mathbf{T}$ HE internal flow dynamics of a solid propellant rocket motor (SRM) is very complex. The combustion of the solid propellant occurs in a thin, high temperature layer between the propellant grain and the main flow cavity, known as the combustion interface. This topologically complex surface evolves as the propellant burns. Moreover, the flow of the propellant products from the combustion chamber through the nozzle, and the plume of the rocket, is a high speed, high temperature, multi-phase, chemically reactive, turbulent flow. For spin stabilized rockets, it is inherently three-dimensional due to rocket rotation, turbulent flow, mass injection, and propellant grain geometry. Propellant burning rates must be controlled to avoid catastrophic failure and inert particles are often added to the propellant to enhance burning stability. The particles, however, can have detrimental effects on the rocket motor, causing excessive erosion of the throat and nozzle, and altering the effective thrust and choking of the rocket motor.

Modern numerical methods are a potential tool for studying the characteristics of the core flows of SRMs, as well as aiding rocket motor design and optimization. Recent examples of the application of numerical methods to the modelling of SRM flows include the studies

\footnotetext{
*Ph.D. Candidate, j.sachdev@utoronto.ca

${ }^{\dagger}$ Assistant Professor, Senior Member AIAA, groth@utias. utoronto.ca

‡Professor, gottlieb@utias.utoronto.ca
}

by Daniel et al., ${ }^{1}$ Orbekk, ${ }^{2}$ Ciucci and co-workers, ${ }^{3}$ Sabnis, ${ }^{4}$ and at the Center for Simulation of Advanced Rockets. ${ }^{5,6}$ The development and description of a parallel solution-adaptive method for predicting axisymmetric, multi-phase SRM core flows is the focus of the present paper. This parallel algorithm has been devised with a view to enabling the computation of complex rocket motor flows on a more routine basis.

In the proposed approach, an Eulerian formulation is used to describe the coupled motion of both the gas and particle phases. A cell-centred upwind finitevolume discretization procedure is used on multi-block quadrilateral meshes to solve the governing partial differential equations in conservation form. Limited linear solution reconstruction and Riemann-solver based flux functions are used to evaluate the numerical fluxes for the gas and particle phases and an explicit multistage time-stepping procedure is used to integrate the governing equations in time. The injection of gas and particle into the core flow from the burning propellant is formulated as a Riemann problem and is solved by an iterative scheme. Adaptive mesh refinement (AMR) is used to accurately resolve multiple solution scales associated with SRM flows. A flexible block-based hierarchical data structure is used to facilitate automatic solution-directed mesh adaptation according to physics-based refinement criteria. This block-based data structure also lends itself naturally to domain decomposition and thereby enables efficient and scalable implementations of the algorithm on distributed-memory multi-processor architectures.

A review of the governing equations is given in the next section. The governing partial differential equa- 
tions are known to be both hyperbolic and degenerate and these properties of the equations are discussed. The proposed numerical solution procedure for the two-phase flow equations on multi-block structured quadrilateral mesh is outlined in the other subsequent sections. The paper concludes by describing numerical results that illustrate the capabilities of the proposed approach for predicting SRM core flows.

\section{Governing Equations}

\section{Gas-Particle Conservation Equations}

An Eulerian formulation is used for the gas and particle phases. The Euler equations of inviscid compressible gas-dynamics are used to describe the gas-phase and a similar set of equations are used to describe the behaviour of the particle phase. The solid particles are assumed to be small and very dense such that the ratio of the particle density, $\rho_{p}$, to gas density, $\rho$, is large $\left(\rho_{p} / \rho \sim 10^{3}\right.$ for typical rocket motors). It then follows that the fraction of the gas-particle mixture volume occupied by the solid particles can be assumed to be very small relative to the volume fraction occupied by the gas phase. Therefore, the particle phase is assumed to be both dilute (negligible volumetric fraction) and disperse (no particle-particle interactions). ${ }^{7}$ There is, however, a strong interaction between the heavy solid particles and the gas due to momentum transfer (drag) between the two phases. Heat transfer between the phases also occurs for cases where the phases have different temperatures. The set of partial differential equations governing the coupled motion of the disperse gas-particle flow for an axisymmetric coordinate system is given by

$$
\frac{\partial \mathbf{U}}{\partial t}+\frac{\partial \mathbf{F}_{\mathbf{r}}}{\partial r}+\frac{\partial \mathbf{F}_{\mathbf{z}}}{\partial z}+\frac{\mathbf{S}}{r}=\mathbf{P}
$$

where $\mathbf{U}$ represents the conserved variable solution vector,

$$
\mathbf{U}=\left[\rho, \rho v_{r}, \rho v_{z}, E, \sigma_{p}, \sigma_{p} u_{r}, \sigma_{p} u_{z}, E_{p}\right]^{T},
$$

$\sigma_{p}$ is the mass concentration of the solid particles, $v_{r}$ and $v_{z}$ are the radial and axial components of the gas velocity $\mathbf{v}, u_{r}$ and $u_{z}$ are the radial and axial components of the particle velocity $\mathbf{u}$, and $E$ and $E_{p}$ are the total energy per unit volume of the gas and particle phases. The vectors $\mathbf{F}_{\mathbf{r}}$ and $\mathbf{F}_{\mathbf{z}}$ are the flux vectors in the $r$ and $z$ directions respectively,

$$
\mathbf{F}_{\mathbf{r}}=\left[\begin{array}{c}
\rho v_{r} \\
\rho v_{r}^{2}+p \\
\rho v_{r} v_{z} \\
v_{r}(E+p) \\
\sigma_{p} u_{r} \\
\sigma_{p} u_{r}^{2} \\
\sigma_{p} u_{r} u_{z} \\
u_{r} E_{p}
\end{array}\right], \quad \mathbf{F}_{\mathbf{z}}=\left[\begin{array}{c}
\rho v_{z} \\
\rho v_{r} v_{z} \\
\rho v_{z}^{2}+p \\
v_{z}(E+p) \\
\sigma_{p} u_{z} \\
\sigma_{p} u_{r} u_{z} \\
\sigma_{p} u_{z}^{2} \\
u_{z} E_{p}
\end{array}\right]
$$

The vectors $\mathbf{S}$ and $\mathbf{P}$ represent the sources due to the axisymmetric flow geometry and the gas-particle interaction,

$$
\begin{gathered}
\mathbf{S}=\left[\rho v_{r}, \rho v_{r}^{2}, \rho v_{r} v_{z}, v_{r}(E+p)\right. \\
\left.\sigma_{p} u_{r}, \sigma_{p} u_{r}^{2}, \sigma_{p} u_{r} u_{z}, u_{r} E_{p}\right]^{T} \\
0 \\
\mathbf{P}=\left[\begin{array}{c}
\sigma_{p} \\
\tau_{v}^{v} \\
-\frac{\sigma_{p}}{\tau_{v}}\left(v_{r}-u_{r}\right) f\left(\operatorname{Re}_{\mathrm{p}}\right) \\
-\frac{\sigma_{p}}{\tau_{v}}(\mathbf{v}-\mathbf{u}) \cdot \mathbf{u} f\left(\operatorname{Re}_{\mathrm{p}}\right)-\frac{\sigma_{p} c_{p}}{\tau_{T}}\left(T-T_{p}\right) \\
0 \\
\frac{\sigma_{p}}{\tau_{v}}\left(v_{r}-u_{r}\right) f\left(\operatorname{Re}_{\mathrm{p}}\right) \\
\frac{\sigma_{p}}{\tau_{v}}\left(v_{z}-u_{z}\right) f\left(\operatorname{Re}_{\mathrm{p}}\right) \\
\frac{\sigma_{p}}{\tau_{v}}(\mathbf{v}-\mathbf{u}) \cdot \mathbf{u} f\left(\operatorname{Re}_{\mathrm{p}}\right)+\frac{\sigma_{p} c_{p}}{\tau_{T}}\left(T-T_{p}\right)
\end{array}\right]
\end{gathered}
$$

The relaxation times associated with the momentum (drag) and heat transfer between the two phases are

$$
\tau_{v}=\frac{m_{p}}{3 \pi d_{p} \mu}, \quad \tau_{T}=\frac{m_{p} c_{p}}{2 \pi d_{p} \kappa},
$$

where $m_{p}$ is the particle mass, $d_{p}$ is the particle diameter, and $c_{p}, \mu$, and $\kappa$, are the gas specific heat at constant pressure, viscosity, and thermal conductivity, respectively. Note that the ratio of the relaxation times can be related to the Prandtl number of the gas, $\tau_{T} / \tau_{v}=\frac{3}{2} \operatorname{Pr}$. The function $f\left(\operatorname{Re}_{\mathrm{p}}\right)$ is a correction to the Stokes drag law

$$
\mathrm{C}_{\mathrm{D}}=\frac{24}{\operatorname{Re}_{\mathrm{p}}} f\left(\operatorname{Re}_{\mathrm{p}}\right)=\frac{24}{\operatorname{Re}_{\mathrm{p}}}\left(1+\frac{1}{6} \operatorname{Re}_{\mathrm{p}}{ }^{\frac{2}{3}}\right) .
$$

This drag coefficient is valid for flow situations satisfying $\operatorname{Re}_{\mathrm{p}}<1000$, where $\operatorname{Re}_{\mathrm{p}}=\left(\rho d_{p} / \mu\right)|\mathbf{v}-\mathbf{u}|$. The ratio of the particle density to gas density is assumed to be large enough such that the Basset history, fluid acceleration, and added mass forces can be neglected. ${ }^{7}$

The gas-phase is taken to be both thermally and calorically perfect and the total energies of the two phases are then given by

$$
\begin{aligned}
& E=\frac{p}{(\gamma-1)}+\frac{\rho}{2}(\mathbf{v} \cdot \mathbf{v}), \\
& E_{p}=\sigma_{p} c_{m} T_{p}+\frac{\sigma_{p}}{2}(\mathbf{u} \cdot \mathbf{u}),
\end{aligned}
$$

where $\gamma=c_{p} / c_{v}$ is the ratio of the specific heats for the gas, $p$ is the gas phase pressure, $c_{m}$ is the specific heat of the particles, and $T_{p}$ is the particle temperature. The ideal gas law provides a relationship between the gas pressure, $p$, and temperature, $T$,

$$
p=\rho \mathrm{R} T=\frac{\rho a^{2}}{\gamma},
$$

where $a=\sqrt{\gamma \mathrm{RT}}$ is the sound speed and $\mathrm{R}$ is the gas constant. 


\section{Degenerate Hyperbolic System}

It has been shown by previous authors that the set of equations governing disperse gas-particle flows is both hyperbolic and degenerate. ${ }^{8,9}$ A brief summary of the cause and implications of the degeneracy now follows.

In order to assess the hyperbolicity and degeneracy of the two-phase flow equations of interest, consider the one-dimensional, weak conservation, form of the equations given above:

$$
\frac{\partial \mathbf{U}}{\partial t}+\frac{\partial}{\partial x} \mathbf{F}(\mathbf{U})=\mathbf{P}(\mathbf{U}),
$$

where $\mathbf{U}=\left[\rho, \rho v, E, \sigma_{p}, \sigma_{p} u, E_{p}\right]^{T}, \mathbf{F}(\mathbf{U})$ is the onedimensional flow state vector of conserved variables and $\mathbf{F}(\mathbf{U})$, and $\mathbf{P}(\mathbf{U})$ are the flux and phaseinteraction source vectors, respectively. This is an inhomogeneous hyperbolic system. In the "frozen" limit, the characteristic times scales of the particle drag and heat transfer, $\tau_{v}$ and $\tau_{T}$, can be assumed to be large relative to the differences in velocities and temperatures of the two phases such that the phaseinteraction terms can be neglected. In this limit, the equations governing the gas and particle phases decouple. An eigenanalysis of the system in the frozen limit provides the set of real eigenvalues, $\lambda_{k}$,

$$
\lambda_{1}=v-a, \quad \lambda_{2}=v, \quad \lambda_{3}=v+a, \quad \lambda_{4,5,6}=u,
$$

indicating the hyperbolic nature of the equations. Note the three repeated eigenvalues associated with the particle phase. The right eigenvectors corresponding to the frozen-limit eigenvalues are

$$
\left(\mathbf{r}_{1}, \mathbf{r}_{2}, \mathbf{r}_{3}, \mathbf{r}_{4}, \mathbf{r}_{5}\right)=\left[\begin{array}{ccccc}
1 & 1 & 1 & 0 & 0 \\
-a / \rho & 0 & a / \rho & 0 & 0 \\
a^{2} & 0 & a^{2} & 0 & 0 \\
0 & 0 & 0 & 1 & 0 \\
0 & 0 & 0 & 0 & 0 \\
0 & 0 & 0 & 0 & 1
\end{array}\right]
$$

This is an incomplete set of eigenvectors as only five linearly independent eigenvectors can be found for the six eigenvalues. An eigenvector associated with the $\lambda=u$ eigenvalue is missing. Because of this degeneracy in the eigensystem, the set of hyperbolic conservation equations governing disperse gas-particle flows is said to be degenerate.

The preceding degeneracy is a direct result of the assumptions that the particle volume fraction is negligible and that the effects of inter-particle collisions are not important. The lack of particle collisions means that there are no normal surface forces produced by the random motion of the particles themselves and hence there is no pressure-like term in the particle momentum and energy equations. Physically, the main implications of the degeneracy are two-fold. First, particle vacuums can exist as there are no direct pressure forces to drive the particles from regions of high concentrations to those with lower concentrations. Moreover, as interactions between particles have been neglected, the paths of particles can cross. Faster moving particles can freely over-take and pass slower moving particles without being subject to any particleparticle interaction forces.

The degenerate nature of the two-phase flow equations must be accounted for when designing a Godunov-type numerical scheme for their solution. Although, Lagrangian modelling methods ${ }^{10}$ can readily deal with particle trajectories that cross, Eulerian finite-volume methods can produce inaccurate and physically incorrect numerical solutions. This is a result of insufficient characteristic fields from which to reconstruct the solution and therefore solution information and accuracy can be lost. The solution algorithm proposed here will make use of the Riemann solver of Saurel et al. ${ }^{8}$ that allows particle paths to cross, however, flows involving the compression of the particle phase are problematic.

\section{Dispersion Analysis}

A dispersion analysis can be used to provide a better understanding of the dynamic behaviour and wave structure of the coupled gas-particle flow equations. An analysis of the one-dimensional form of these equations is considered here. A similar analysis was carried out by Marble. ${ }^{11}$ The construction of the equilibrium, frozen, and non-equilibrium dispersion relationships follows the procedure outlined by Groth et al. ${ }^{12}$

The dispersive wave properties of the one-dimensional form of the gas-particle flow equations given by (11) can be assessed by considering the linearized primitive form of these equations given by

$$
\frac{\partial \mathbf{W}}{\partial t}+\mathbf{A}_{0} \frac{\partial \mathbf{W}}{\partial x}=\mathbf{Q}_{0} \mathbf{W}
$$

where $\mathbf{W}=\left[\rho, v, T, \sigma_{p}, u, T_{p}\right]^{T}$ is the primitive solution vector and the matrices $\mathbf{A}_{\mathbf{0}}$ and $\mathbf{Q}_{\mathbf{0}}$ are the linearized coefficient matrix and phase-interaction source Jacobian determined by linearizing the primitive form of the equations about a quiescent (stationary) equilibrium state $\mathbf{W}_{\mathbf{0}}=\left[\rho_{0}, 0, T_{0}, \sigma_{p 0}, 0, T_{0}\right]^{T}$. For initial value problems and solutions of the form $\mathbf{W}=$ $\exp [i(\omega t-\xi x)]$, the differential wave operator for the linearized equations is defined by the the eigenvalue problem given by $\operatorname{det}\left(i \omega \mathbf{I}-i \xi \mathbf{A}_{\mathbf{0}}-\mathbf{Q}_{\mathbf{0}}\right)=0$ where the temporal frequency $\omega$ can have real and imaginary components, $\omega=\omega_{R}+i \omega_{I}$, whereas the spatial frequency $\xi$ is real valued. The parameter $\xi$ defines the frequency content of the linearized primitive solution, which varies from the low frequencies for near equilib- 
rium solutions to high frequencies for non-equilibrium solutions in the frozen limit. The condition for the stability of the solutions is $\omega_{I}>0$.

In the equilibrium limit $\left(\tau_{v}, \tau_{T} \rightarrow 0\right)$, the phaseinteraction terms are zero and the particles are constrained by the drag forces and heat transfer to move with gas velocity $(\mathbf{u}=\mathbf{v})$ and have the same temperature $\left(T_{p}=T\right)$. An equilibrium density can be defined by setting $\bar{\rho}=\rho+\sigma_{p}=\rho(1+\chi)$ where $\chi=\sigma_{p} / \rho$ is the particle loading factor and is constant for uniformly distributed particles. The system of equations in the equilibrium limit can then be reduced to three variables, in terms of the primitive variables $\bar{\rho}, v$, and $T$. In this case, the linearized non-dimensional coefficient matrix is given by

$$
\mathbf{A}_{0}=\left[\begin{array}{ccc}
0 & 1 & 0 \\
\overline{\mathrm{R}}_{0} T_{0} / v_{0}^{2} & 0 & \overline{\mathrm{R}}_{0} T_{0} / v_{0}^{2} \\
0 & \overline{\mathrm{R}}_{0} / \bar{c}_{v_{0}} & 0
\end{array}\right] .
$$

where $T_{0}$ is the temperature of the quiescent equilibrium state, $v_{0}$ is a reference velocity, the equilibrium gas constant and specific heat ratios are given by $\overline{\mathrm{R}}_{0}=\bar{c}_{p_{0}}-\bar{c}_{v_{0}}=\mathrm{R} /\left(1+\chi_{0}\right), \bar{c}_{v_{0}}=\left(c_{v}+\chi_{0} c_{m}\right) /\left(1+\chi_{0}\right)$, and $\bar{c}_{p_{0}}=\left(c_{p}+\chi_{0} c_{m}\right) /\left(1+\chi_{0}\right)$, and $\chi_{0}$ is the loading ratio of the reference equilibrium state. Solution of the eigenvalue problem, with $\lambda=\omega / \xi$, provides the equilibrium dispersion relationship,

$$
-\lambda\left[\lambda^{2}-\bar{\gamma} \overline{\mathrm{R}} T_{0} / v_{0}^{2}\right]=0 .
$$

Determination of the roots of this polynomial gives the following non-dimensional equilibrium wave speeds

$$
\lambda_{1}=-\bar{a} / v_{0}, \quad \lambda_{2}=0, \quad \lambda_{3}=\bar{a} / v_{0} .
$$

In the equilibrium limit, the imaginary part of the wave-speed is zero, $\lambda_{I}=0$, which corresponds to nondispersive, coherent wave propagation.

Now consider the linearized equations in the highfrequency or frozen limit. The linearized and nondimensionalized coefficient and source Jacobians are

$$
\begin{aligned}
& \mathbf{A}_{0}= {\left[\begin{array}{cccccc}
0 & 1 & 0 & 0 & 0 & 0 \\
\mathrm{R} T_{0} / v_{0}^{2} & 0 & \mathrm{R} T_{0} / v_{0}^{2} & 0 & 0 & 0 \\
0 & \mathrm{R} / c_{v} & 0 & 0 & 0 & 0 \\
0 & 0 & 0 & 0 & 1 & 0 \\
0 & 0 & 0 & 0 & 0 & 0 \\
0 & 0 & 0 & 0 & 0 & 0
\end{array}\right], } \\
& \mathbf{Q}_{0}=\frac{t_{0}}{\tau_{T}}\left[\begin{array}{cccccc}
0 & 0 & 0 & 0 & 0 & 0 \\
0 & -\frac{3}{2} \chi_{0} \operatorname{Pr} & 0 & 0 & \frac{3}{2} \chi_{0} \operatorname{Pr} & 0 \\
0 & 0 & -\gamma \chi_{0} & 0 & 0 & \gamma \chi_{0} \\
0 & 0 & 0 & 0 & 0 & 0 \\
0 & \frac{3}{2} \operatorname{Pr} & 0 & 0 & -\frac{3}{2} \operatorname{Pr} & 0 \\
0 & 0 & \frac{c_{p}}{c_{m}} & 0 & 0 & -\frac{c_{p}}{c_{m}}
\end{array}\right] .
\end{aligned}
$$

In the frozen limit, the characteristic time scales of the viscous drag and heat transfer terms, $\tau_{v}$ and $\tau_{T}$, are assumed to be large relative to the differences in velocity and temperature such that the source matrix $\mathbf{Q}_{0}$ can be neglected. The dispersion relationship in the frozen limit is found by solving

$$
\operatorname{det}\left(\lambda \mathbf{I}-\mathbf{A}_{0}\right)=-\lambda^{4}\left[\lambda^{2}-\gamma \mathrm{R} T_{0} / v_{0}^{2}\right]=0
$$

where $\lambda=\omega / \xi$. Solution of this polynomial equation yields the non-dimensional frozen wave speeds

$$
\lambda_{1}=-a_{0} / v_{0}, \quad \lambda_{2,4,5,6}=0, \quad \lambda_{3}=a_{0} / v_{0} .
$$

The wave-speeds in the frozen limit show nondispersive, coherent wave propagation since $\lambda_{I}=0$ for each wave, including the four convective waves (three associated with the particle phase) and two acoustic waves (associated with the gas phase).

The dispersion relationship for the general nonequilibrium system can be determined by solving the eigenvalue problem for the characteristic wave-speeds, $\operatorname{det}\left(i \omega \mathbf{I}-i \xi \mathbf{A}_{0}-\mathbf{Q}_{0}\right)=0$. The resulting dispersion relationship is

$$
\lambda^{2}\left(\lambda^{4}-i c_{3} \lambda^{3}-c_{2} \lambda^{2}+i c_{1} \lambda+c_{0}\right)=0
$$

where $\lambda=\omega / \xi$. The spatial frequency, $\xi$, can be expressed as $\xi=2 \pi x_{0} / L=a_{0} t_{0} / a_{0} \tau=t_{0} / \tau$, where $a_{0}=\sqrt{\gamma \mathrm{R} T_{0}}, t_{0}$ is a reference time scale, and $L=a_{0} \tau$ is the wavelength of the solution. The frozen and equilibrium limits are defined by high and low frequency solution limits (i.e., $\tau \rightarrow 0$ and $\tau \rightarrow \infty$, respectively). The coefficients $c_{3}, c_{2}, c_{1}$, and $c_{0}$ are given by

$$
\begin{aligned}
& c_{3}=\left(1+\chi_{0}\right)\left(\frac{\tau}{\tau_{v}}+\gamma \frac{\bar{c}_{v_{0}}}{c_{m}} \frac{\tau}{\tau_{T}}\right), \\
& c_{2}=\frac{\gamma \mathrm{R} T_{0}}{v_{0}^{2}}+\gamma\left(1+\chi_{0}\right)^{2} \frac{\bar{c}_{v_{0}}}{c_{m}} \frac{\tau}{\tau_{v}} \frac{\tau}{\tau_{T}}, \\
& c_{1}=\frac{\gamma \mathrm{R} T_{0}}{v_{0}^{2}}\left(\frac{\tau}{\tau_{v}}+\left(1+\chi_{0}\right) \frac{\bar{c}_{p_{0}}}{c_{m}} \frac{\tau}{\tau_{T}}\right), \\
& c_{0}=\left(1+\chi_{0}\right) \frac{\gamma \mathrm{R} T_{0}}{v_{0}^{2}} \frac{\bar{c}_{p_{0}}}{c_{m}} \frac{\tau}{\tau_{v}} \frac{\tau}{\tau_{T}} .
\end{aligned}
$$

In the general non-equilibrium case, the nondimensional wave speeds, $\lambda$, can have real and imaginary components, $\lambda=\lambda_{R}+i \lambda_{I}$, characteristic of dispersive wave propagation. Analytic expressions for the non-dimensional wave speeds cannot be found; however, it is instructive to investigate the dispersion relationship for the non-equilibrium system by numerically calculating the waves speeds as a function of the solution frequency. For these purposes, the physical properties of the gas and solid phase produced by the combustion of the solid propellant are consistent with a non-aluminized composite propellant composed of 


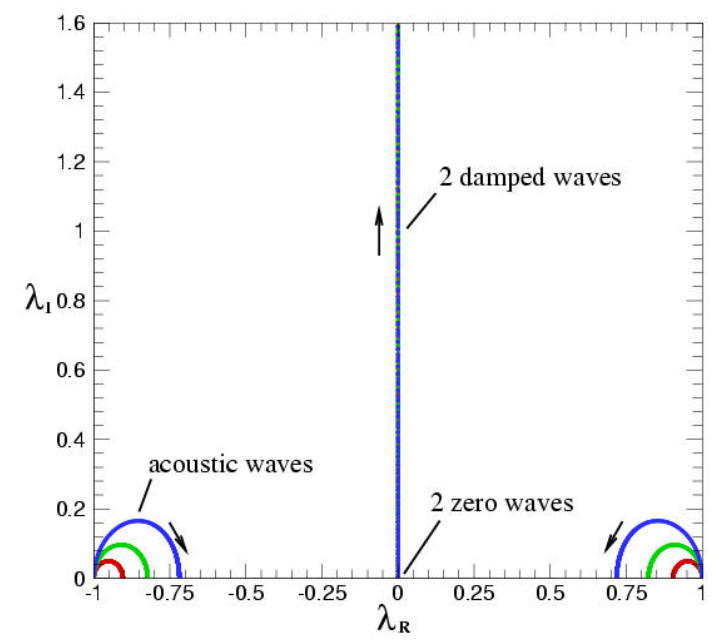

Fig. 1 Dispersion relationship with various loading factors: $\cdot \chi=0.20, \cdot \chi=0.42, \cdot \chi=0.82$.

$80 \%$ oxidizer (ammonium perchlorate, AP) and $20 \%$ fuel (hydroxyl-terminated polybutadiene, HTPB).

The effect of the frequency content of the linearized solution on the dispersive wave behaviour is shown in Figure 1 for various loading ratios, $\chi_{0}$. The arrows indicate the direction of change from the frozen limit (high frequency) to the equilibrium limit (low frequency). Four of the solution modes are undamped in the low frequency equilibrium limit. Two of these, the acoustic modes, have finite damping for more intermediate solution frequencies and become undamped once again as the non-equilibrium frozen limit is reached. The other two modes, remain undamped for all solution frequencies and are associated with convective transport of the gas and particle phase densities. The remaining two modes are also convective modes associated with the transport of differences in the velocities and temperatures of the two phases. They are highly attenuated in the equilibrium limit. This is confirmed by the equilibrium wave solution determined previously, equation (17), where the gas and solid phases have the same velocity and temperature. In the frozen limit, damping of these last two modes approaches zero. Note also the interleaving of the non-equilibrium wave speeds between the frozen and equilibrium limits. This ensures the stability of the solution and is an important feature that must be considered when developing upwind numerical schemes for the general solution of the two-phase flow equations.

The attenuation rates for all of the modes as a function of the solution wave length are plotted in Figure 2. This plot shows that the two purely damped modes quickly become highly damped as the wavelength increases (frequency decreases). The zero damping of the acoustic modes in both the equilibrium and froze limit is clearly depicted.

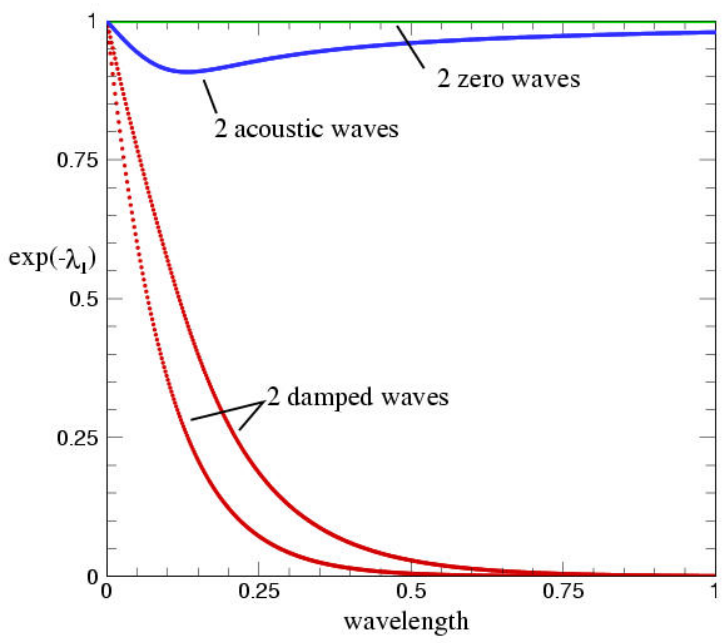

Fig. 2 Wave attenuation rates as a function of solution wavelength, $L=a_{0} \tau$, for a loading factor of $\chi=0.42$.

\section{Numerical Method}

\section{Finite Volume Scheme}

In this work, an explicit higher-order Godunov-type finite-volume scheme is used to solve the gas-particle equations. Upwind finite-volume schemes for the gasdynamic equations were originally introduced by Godunov. ${ }^{13}$ Application and development of these schemes for the gas dynamic equations has been well documented in literature. Refer to the textbook by Hirsch $^{14}$ for details.

In this finite-volume approach, the governing equations are integrated over quadrilateral cells of a structured multi-block quadrilateral mesh. The integration over cell $i$ is given by

$$
\frac{\mathrm{d} \mathbf{U}_{i}}{\mathrm{~d} t}=-\frac{1}{A_{i}} \sum_{\text {faces }} \overrightarrow{\mathbf{F}} \cdot \overrightarrow{\mathbf{n}} \Delta \ell-\frac{\mathbf{S}_{i}}{r}+\mathbf{P}_{i},
$$

where $\overrightarrow{\mathbf{F}}=\left[\mathbf{F}_{\mathbf{r}}, \mathbf{F}_{\mathbf{z}}\right], A_{i}$ is the area of cell $i$ and $\Delta \ell$ and $\mathbf{n}$ are the length of the cell face and unit vector normal to the cell face or edge, respectively.

The numerical fluxes at the faces of each cell are determined from the solution of a Riemann problem. Given the left and right solution states, $\mathbf{U}_{l}$ and $\mathbf{U}_{r}$, at the cell interfaces, the numerical flux is given by

$$
\overrightarrow{\mathbf{F}} \cdot \overrightarrow{\mathbf{n}}=\mathcal{F}\left(\mathbf{U}_{l}, \mathbf{U}_{r}, \overrightarrow{\mathbf{n}}\right) .
$$

where $\mathcal{F}$ is evaluated by solving a Riemann problem in a direction defined by the normal to the face, $\mathbf{n}$, with initial data $\mathbf{U}_{l}$ and $\mathbf{U}_{r}$. The left and right solution states are determined using piece-wise linear solution reconstruction using the least squares limited reconstruction procedure developed by Barth. ${ }^{15}$

Frozen flow conditions are assumed for the solution of the Riemann problem. As was shown in the previous 
section, the phase interactions terms vanish and the gas and particle phases fully decouple in the frozen flow limit. Hence, separate Riemann problems and solutions can be formulated for the two phases. For the gas phase, the Roe flux function, ${ }^{16}$ HLLE flux function, ${ }^{17}$ the HLLC flux function, ${ }^{18}$ and the exact Riemann solver of Gottlieb and Groth ${ }^{19}$ have all been implemented. For the particle phase, the Riemann solver proposed by Saurel et al. ${ }^{8}$ has been implemented and is discussed in the next section.

For time-accurate calculations, predictor-corrector and fourth order Runge-Kutta time-marching methods are used to integrate the set of ordinary differential equation that result from the spatial discretization of the governing equations. The optimally-smoothing multi-stage schemes developed by van Leer et al. ${ }^{20}$ are adopted for steady-state calculations.

\section{Particle Phase Riemann Solver}

Determination of the flux values at cell interfaces for the particle-phase is complicated by the degeneracy of the governing flow equations described above. A particle-phase Riemann solver was proposed by Saurel et $a l .{ }^{8}$ and its limitations are discussed here.

Given the left and right particle phase solution states, Saurel et al. identified and used three expansion wave configurations and three compression wave configurations to construct solutions to the Riemann problem. In all three of the compressive cases, the interface solution compromises the actual physics of the particle motions. For the mildly compressive cases where one solution state is catching up with the other, the solution information carried by the overtaken particles is lost. In reality, this solution information should be retained. For the more strongly compressive case, the two waves (populations of particles) should simply pass through each other. Instead, the particle concentrations are directly summed and density weighted averages are assigned to the particle velocity and temperature. Although this averaging procedure provides the correct average state for the combined left and right moving particle populations, it fails to recognize the presence of the two oppositely moving populations and this solution content is lost and not retained in the numerical approximations to the solution. This can lead to the unphysical results and difficulties near solid boundaries as described by Saurel et al. and by Slater and Young ${ }^{9}$ and is a basic limitation of single-velocity Eulerian formulations for describing particle phase motions. Lagrangian formulations, which track and solve for the individual particle motions, can account for multiple particle trajectories within a computational cell and thereby avoid the problems associated with degeneracy of the Eulerian formulations. However, La- grangian methods generally require significantly more computing resources than an Eulerian approach, in terms of both memory and computing time. This is particularly true for two-phase particle-laden flows where the phases are strongly coupled and have a significant affect on each other. Efficient and scalable parallel implementations of Lagrangian particle tracking formulations are also difficult to achieve.

\section{Burning Surface Boundary Condition}

The combustion of the solid propellant of the rocket motor occurs in a thin, high temperature layer between the solid propellant and the main flow cavity, known as the combustion interface. This layer is assumed to be small relative to the diameter of the rocket motor and large relative to the product of the propellant product velocities and chemical reaction relaxation times such that the finite-rate nature of the chemical reactions can be neglected and the injected gas-particle products can be assumed to be in chemical equilibrium. Boundary conditions for a regressing burning surface that injects gas-particle products into the flow cavity is formulated in terms of a Riemann problem and used here to specify boundary data at the surface of the burning propellant. The treatment of the burning propellant boundary is very similar in spirit to the methods proposed by Gottlieb and Groth ${ }^{21}$ for imposing boundary data at a variety of flow boundaries based on the solution of Riemann problems.

For the current analysis, the burning rate is computed by the application of the pressure dependent empirical St. Robert relation,

$$
\rho_{b s} v_{b s}=\left(1-\alpha_{s}\right) \rho_{s} r_{b s}=\left(1-\alpha_{s}\right) \rho_{s} \beta p_{b s}^{n}
$$

where $\rho_{b s}, v_{b s}$, and $p_{b s}$ are the density, normal velocity, and pressure of the gas injected from the burning surface and $\rho_{s}$ is the solid propellant density. The burning rate, $r_{b s}$, is the same as the injected gas velocity and the burning rate constants $\beta$ and $n$ are functions of the chemical composition of the solid and the adiabatic flame temperature, $T_{f}$. The mass fraction of solid particles in the propellant is given by $\alpha_{s}$. Note that the speed of the propagating surface is $-r_{b s}$. Erosive burning effects are not included in the present work.

The wave solution for the burning surface Riemann problem is shown in Figure 3. There are two waves: a contact surface and a second wave which can be a shock or a rarefaction wave. The resulting system of equations, the shock/rarefaction equations and the burning rate equation (25), is nonlinear and implicit in the unknown burning surface pressure or normal velocity, $p_{b s}$ and $v_{b s}$. A Newton iteration scheme is developed to solve this system with the burning surface pressure as the iterate. Propagation of the burning 
surface can be accounted for through an application of a Galilean transformation to a frame that the surface is stationary. In this frame, the right-state velocity is given by $v_{x r}^{s}=v_{x r}+r_{b s}$. The burning surface velocity in the stationary frame, $v_{b s}^{s}$, can now be determined by the iterative scheme.

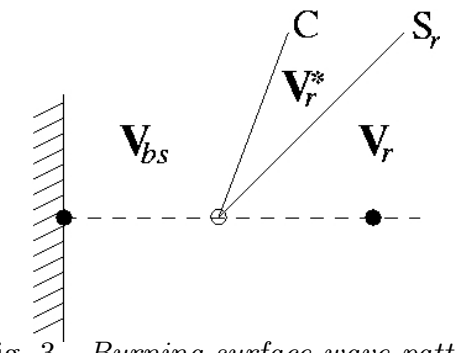

Fig. 3 Burning surface wave pattern.

\section{Block-Based Adaptive Mesh Refinement}

Adaptive mesh refinement techniques which automatically adapt the computational grid to the solution of the governing partial differential equations can be very effective in treating problems with disparate length scales. Following the approach developed by Groth et al. for computational magnetohydrodynamics, ${ }^{22,23}$ a flexible block-based hierarchical data structure has been developed and is used in conjunction with the finite-volume scheme described above to facilitate automatic solution-directed mesh adaptation on multiblock quadrilateral mesh according to physics-based refinement criteria.

In this work, the governing equations are integrated to obtain area-averaged solution quantities within quadrilateral computational cells and these cells are embedded in structured blocks consisting of $N_{x} \times N_{y}$ cells, where $N_{x}$ and $N_{y}$ are even, but not necessarily equal integers. Solution data associated with each block are stored in indexed array data structures and it is therefore straightforward to obtain solution information from neighboring cells within blocks. Mesh adaptation is accomplished by the dividing and coarsening of appropriate solution blocks. In regions requiring increased cell resolution, a "parent" block is refined by dividing itself into four "children" or "offspring". Each of the four quadrants or sectors of a parent block becomes a new block having the same number of cells as the parent and thereby doubling the cell resolution in the region of interest. This process can be reversed in regions that are deemed over-resolved and four children are coarsened into a single parent block. The mesh refinement is constrained such that the grid resolution changes by only a factor of two between adjacent blocks and the minimum resolution is not less than that of the initial mesh. Standard multigridtype restriction and prolongation operators are used to evaluate the solution on all blocks created by the coarsening and division processes, respectively. Al-

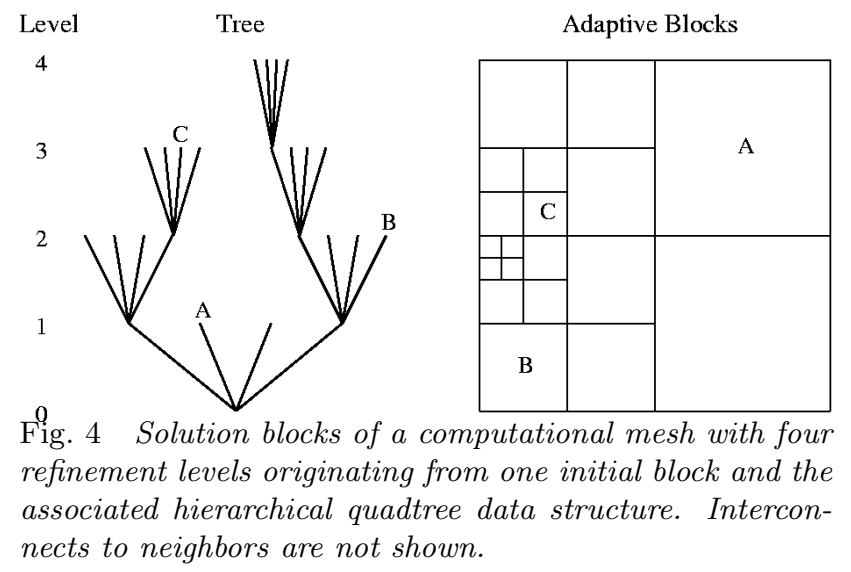

though several approaches are possible, for this study, the coarsening and division of blocks are directed using multiple physics-based refinement criteria. ${ }^{24}$

In order that the solution algorithm for the multiphase flow equations can be applied to all blocks in a more independent manner, some solution information is shared between adjacent blocks having common interfaces. This information is stored in an additional two layers of overlapping "ghost" cells associated with each block. At interfaces between blocks of equal resolution, these ghost cells are simply assigned the solution values associated with the appropriate interior cells of the adjacent blocks. At resolution changes, restriction and prolongation operators, similar to those used in block coarsening and division, are employed to evaluate the ghost cell solution values. Within the AMR approach, additional inter-block communication is also required at interfaces with resolution changes to strictly enforce the flux conservation properties of the finite-volume scheme. ${ }^{25}$ In particular, the interface fluxes computed on more refined blocks are used to correct the interface fluxes computed on coarser neighboring blocks and ensure the solution fluxes are conserved across block interfaces.

A hierarchical tree-like data structure with multiple "roots", multiple "trees", and additional interconnects between the "leaves" of the trees is used to keep track of mesh refinement and the connectivity between solution blocks. This interconnected "forest" data structure is depicted in Figure 4. The blocks of the initial mesh are the roots of the forest which are stored in an indexed array data structure. Associated with each root is a separate "quadtree" data structure that contains all of the blocks making up the leaves of the tree created from the original parent blocks during mesh refinement. Each grid block corresponds to a node of the tree. Traversal of the multi-tree structure by recursively visiting the parents and children of solution blocks can be used to determine block connectivity. However, in order to reduce overhead associated with accessing solution information from adjacent blocks, 


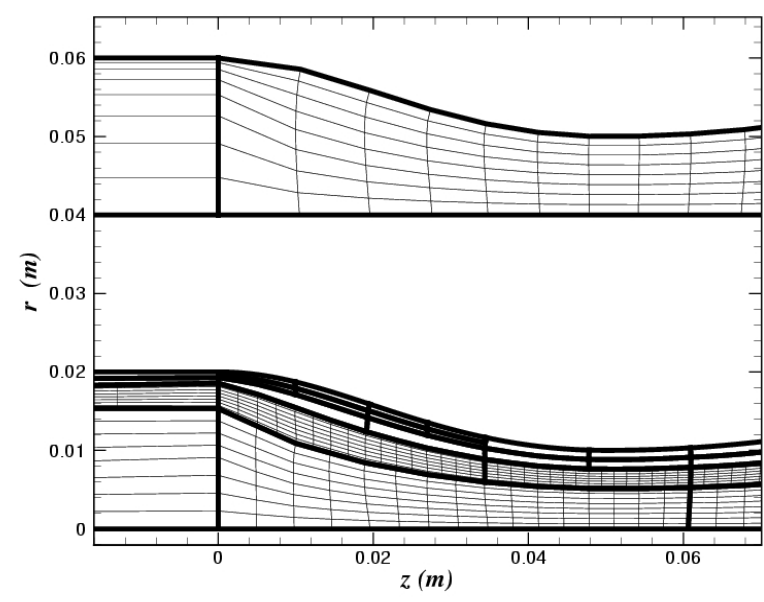

Fig. 5 Illustration of AMR for two-dimensional multiblock quadrilateral mesh showing initial 2-block mesh and final 38-block refined mesh after four levels of refinement.

the neighbors of each block are computed and stored, providing direct interconnects between blocks in the hierarchical data structure that are neighbors in physical space. One of the advantages of the hierarchical quadtree data structure is that it readily permits local mesh refinement at point in a calculation. Local modifications to the multi-block mesh can be performed without re-gridding the entire mesh and re-calculating all solution block connectivities.

An example illustrating the adaptation of a twodimensional multi-block quadrilateral mesh for a simplified rocket motor core flow geometry is shown in Figure 5. The figure shows an initial grid consisting of two blocks and 256 cells $(16 \times 8$ cell blocks were used $)$ and a refined mesh derived from the initial mesh by applying four levels of refinement. The resulting refined mesh consists of 38 blocks and 4,864 cells. The solution block boundaries (thick lines) and computational cells (thin lines) for both mesh are depicted in the figure. Note that each level of refinement in the grid introduces cells that are typically smaller by a factor two in each spatial dimension. Practical calculations may have 10-15 levels of refinement. In the case of 15 levels of refinement, the finest cells in the mesh are more than 32,000 $\left(2^{15}\right)$ times smaller than the coarsest cells. Note also that the initial mesh stretching, in this case applied to the mesh in the radial direction, is retained by the mesh refinement procedure such that the refined blocks and the cells within them are clustered near the upper boundary of the mesh. Use of cell stretching and cell clustering in the initial mesh enables anisotropic refinement of the multi-block grid, which will be particularly important for resolving boundary and shear layers in subsequent studies of viscous core flows in rocket motors.

\section{Parallel Implementation}

Although the block-based AMR approach described above is somewhat less flexible and incurs some inefficiencies in solution resolution as compared to a cellbased approaches (i.e., for the same solution accuracy, generally more computational cells are introduced in the adapted grid), the block-based method offers many advantages over cell-based techniques when parallel implementation of the solution algorithm is considered and computational performance issues are taken into account. In particular, the multi-block quadrilateral mesh and quadtree data structure lends itself naturally to domain decomposition and thereby enables efficient and scalable implementations of the solution algorithm for the two-phase flow equations on distributed-memory multi-processor architectures.

A parallel implementation of the block-based AMR scheme has been developed using the $\mathrm{C}++$ programming language and the MPI (message passing interface) library. Use of these standards greatly enhances the portability of the computer code and has enabled very good parallel performance. Domain decomposition is carried out by merely farming the solution blocks out to the separate processors, with more than one block permitted on each processor. A simple stack is used to keep track of available (open) processors. For homogeneous architectures with multiple processors all of equal speed, an effective load balancing is achieved by exploiting the self-similar nature of the solution blocks and simply distributing the blocks equally amongst the processors. In doing so, all blocks are treated equally and, currently, no use is made of the hierarchical data structure nor grid partitioning techniques to preferentially place neighboring blocks on the same processors. With 10 blocks per processor, the maximum load imbalance attained by this simple block distribution procedure is less than $10 \%$ (near perfect load balancing is achieved if the number of block is a exact multiple of the number of available processors). For heterogeneous parallel machines, such as a network of workstations and computational grids, a weighted distribution of the blocks can be adopted to preferentially place more blocks on the faster processors and less blocks on the slower processors.

In order to carry out mesh refinement and inter-block communication, a complete copy of the hierarchical quadtree data structure is stored on each processor. This is possible because, unlike cell-based unstructured meshing techniques, the block-based tree data structure is not overly large. The structure need only retain the connectivity between the solution blocks as opposed to a complete map of the cell connectivity required by general unstructured mesh procedures. Inter-processor communication is mainly associated 


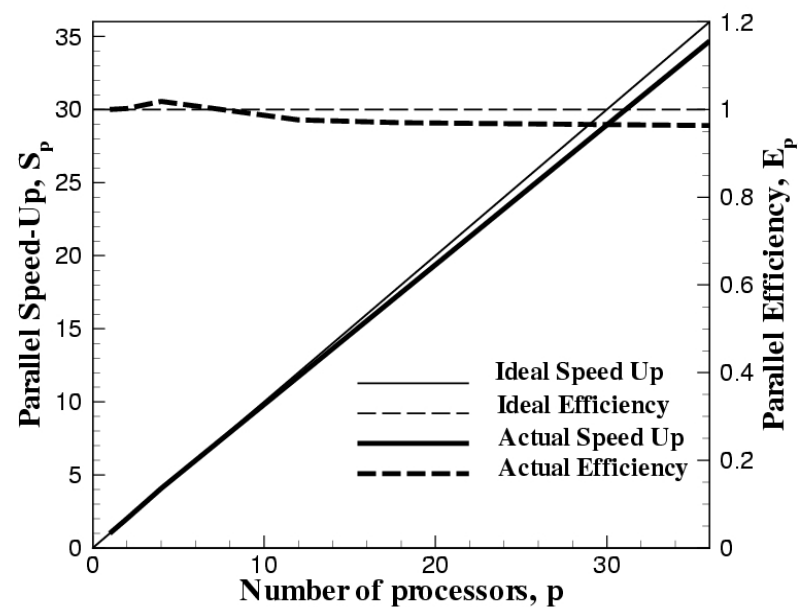

Fig. 6 Parallel scaled speed-up, $S_{p}$, and Parallel efficiency, $E_{p}$, for a fixed-size problem using up to 36 processors.

with block interfaces and involves the exchange of ghost-cell solution values and conservative flux corrections at every stage of the multi-stage time integration procedure. Message passing of the ghost-cell values and flux corrections is performed in an asynchronous fashion with gathered wait states and message consolidation, and as such, typically amounts to less than $3-5 \%$ of the total processor time.

Implementation of the algorithm has been carried out on Beowulf cluster of 4-way Hewlett-Packard ES40 and ES45 servers with a total of 104 Alpha processors. A low-latency Myrinet network and switch is used to interconnect the servers in the cluster. Some initial estimates of the parallel performance and scalability of the proposed method on this parallel architecture are shown in Figure 6 for a fixed size problem involving 36,864 computational cells and 36 solution blocks using up to 36 processors. The figure show both the parallel scaled speed-up, $S_{p}$, given by $S_{p}=\left(t_{p} / t_{1}\right) p$, and the parallel scaled efficiency, $E_{p}$, given by $E_{p}=S_{p} / p$, as a function of the number of processors, $p$, where $t_{p}$ is the total processor time required to solve the problem using $p$ processors and $t_{1}$ is the processor time required to solve the problem using a single processor. It can be seen that the parallel speed-up of the blockbased AMR scheme is linear and exceeds $96 \%$ efficient for up to 36 processors.

\section{Numerical Results}

Numerical SRM core flow results are now described to demonstrate the viability and capabilities of the scheme. The predicted results are presented in Figures $7-11$ for a cylindrical grain rocket motor with a $40 \mathrm{~mm}$ internal radius, a nozzle throat radius of 10 $\mathrm{mm}$, and an internal port radius of $14.5 \mathrm{~mm}$. Predicted results of the type shown in the figures have also been validated quantitatively by comparing the two-dimensional predictions with one-dimensional re-

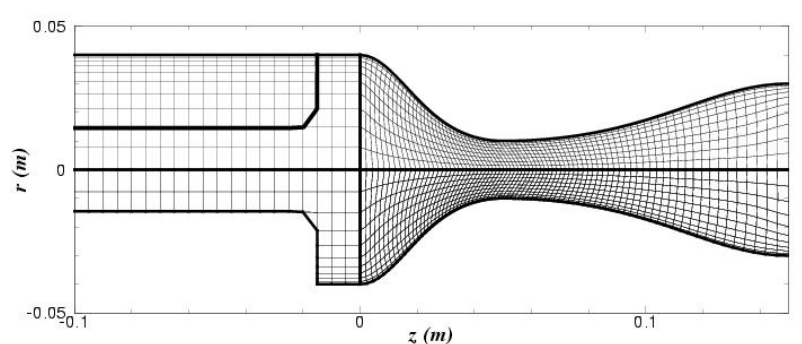

Fig. 7 Initial (upper panel of figure) and adjusted (lower panel of figure) mesh for a cylindrical grain rocket motor.

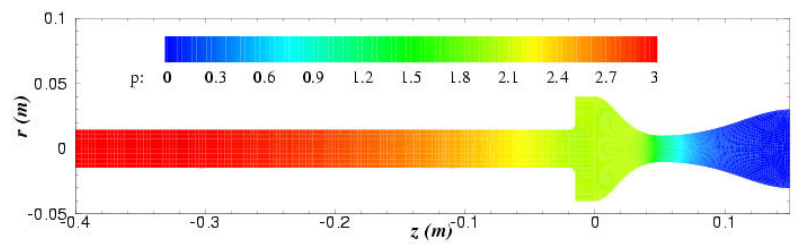

Fig. 8 Predicted propellant gas pressure distribution for a cylindrical grain rocket motor.

sults obtained by Greatrix. ${ }^{26}$ The overall agreement is found to be very good. The propellant grain consists of $95 \%$ AP-HPTB and $5 \%$ inert particles and is considered to be stationary. Future work will include the application of the level set method ${ }^{27}$ to allow for the regression of the propellant interface as it burns. To prepare for this capability, a mesh adjustment algorithm was implemented to adjust an underlying quadrilateral structured mesh to the interface location. Figure 7 demonstrates the grid adjustment algorithm for the rocket motor outlined above. The initial structured multi-block grid is shown in the upper portion where the propellant interface is indicated by a thick solid line and the resulting adjusted grid is shown in the lower portion. Note that triangular cells can be generated from this scheme as can be seen at the propellant corner, however, the structured nature of the structured mesh is maintained.

The burning of the solid propellant leads to a head end pressure in excess of $3 \mathrm{MPa}$, see Figure 8 , and produces sonic flow conditions at the nozzle throat and supersonic outflows in the rocket nozzle with Mach numbers approaching 3.25, see Figure 9. The particlephase concentration through the converging-diverging nozzle is shown in Figure 10 and comparisons of the propellant gas and inert particle axial velocity components is in shown in Figure 11. Both figures also depict the gas and particle streamlines in the upper and lower portions of the figures, respectively. The particle-phase velocity lag relative to the gas-phase velocity after the rapid acceleration through the nozzle is clearly represented in Figure 11 . The particle streamlines show that the particles are unable to expand in the nozzle due to their relative high mass. As expected, a large, low speed recirculation zone is found in the area following the propellant grain and before 


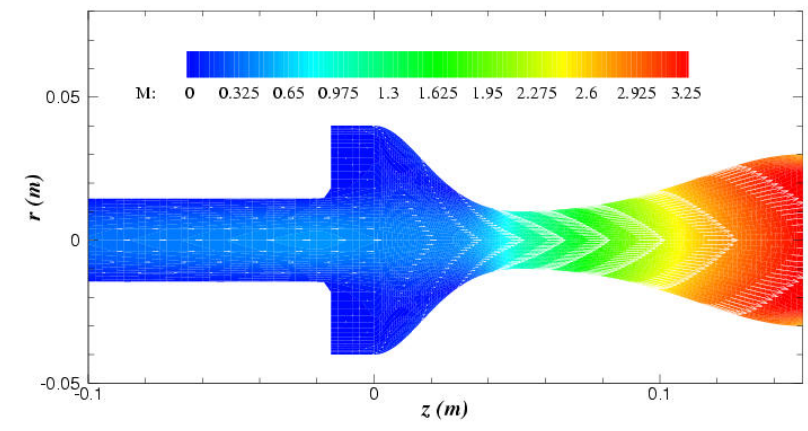

Fig. 9 Predicted propellant gas Mach number distribution and gas-phase velocity vectors for a cylindrical grain rocket motor.

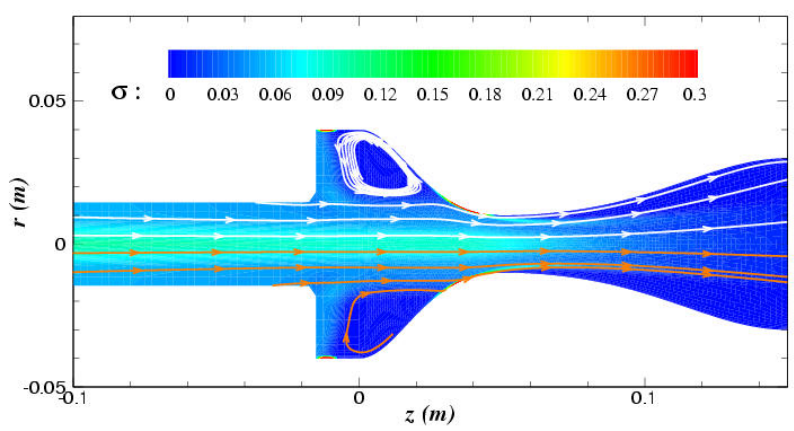

Fig. 10 Predicted particle phase concentration contours with gas-phase (upper panel of figure) and particle-phase streamlines (lower panel of figure) for a cylindrical grain rocket motor.

the converging section of the nozzle. A consequence of this recirculation, is the formation of an area of high particle concentration near the junction between the propellant and the rocket casing since the inert particles are pushed away by the recirculation. A region of high particle concentration can be found at the upper wall of the converging section of the nozzle. A similar result has been found by Vuillot et al. ${ }^{28}$ However, this accumulation of particles maybe somewhat unphysical due to the equation degeneracies and limitations of the particle phase Riemann solver discussed previously. Reflective boundary conditions have been implemented at this boundary, which, due to the degeneracy, leads to an averaging of particle states approaching and leaving the boundary. The boundary condition instead behaves like a slip condition, where the particles will just slide along the wall until become entrained with the gas at the nozzle throat. To avoid this effect, Slater and Young ${ }^{9}$ only consider absorption conditions at solid boundaries. The high particle concentration zone found at the rocket centre-line and the low concentration zone at the walls of the diverging section of the nozzle are are well predicted.

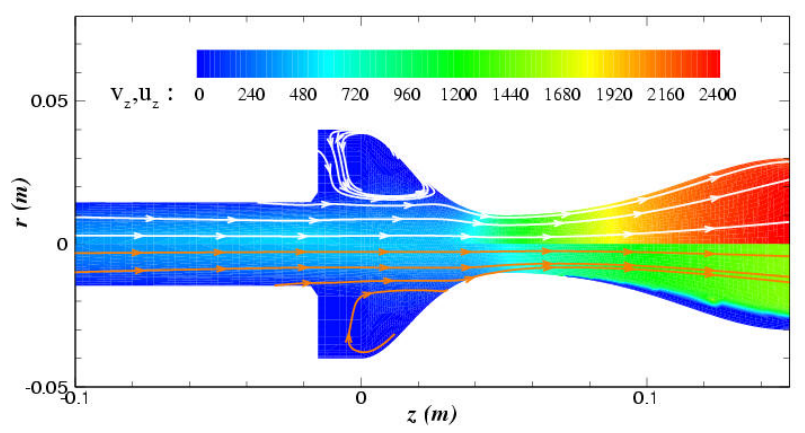

Fig. 11 Axial velocity component and streamlines for the propellant gas phase (upper panel of the figure) and inert particle phase (lower panel of the figure) for a cylindrical grain rocket motor.

\section{Concluding Remarks}

A parallel adaptive mesh refinement scheme has been described for solving the governing equations for multiphase core flows in solid propellant rocket motors. The application of an upwind finite-volume discretization procedure and a parallel block-based AMR strategy has provided a powerful tool for predicting SRM core flows. Parallel implementation has led to high parallelperformance, achieving near-linear scalability. The viability of the method has been demonstrated for a SRM core flow. The limitations of two-phase flow formulation due to the degenerate nature of the governing hyperbolic equations has been identified and examined. Future work will include application of the level set method to allow for a propagating interface as well as extension to a viscous, turbulent core flow and the implementation of multi-grid and Newton-Krylov methods to improve the efficiency of the time integration procedure.

\section{Acknowledgements}

This research was supported by a Premier's Research Excellence Award from the Ontario Ministry of Energy, Science, and Technology and by the Natural Sciences and Engineering Research Council of Canada. Funding for the parallel computing facility used to perform the computations described herein was obtained from the Canadian Foundation for Innovation and Ontario Innovation Trust (CFI Project No. 2169). The authors are very grateful to these funding agencies for this support.

\section{References}

${ }^{1}$ Daniel, E., Basset, T., and Loraud, J. C., "Eulerian approach for unsteady two-phase reactive solid rocket motor flows loaded with aluminum particles," Paper 98-3697, AIAA, 1999. 
${ }^{2}$ Orbekk, E., "Internal flow analysis of a technology demonstrator rocket motor with new CFD code," $\mathrm{Pa}$ per 98-3967, AIAA, July 1998.

${ }^{3}$ Ciucci, A., Iaccarino, G., and Amato, M., "Numerical investigation of 3D two-phase turbulent flows in solid rocket motors," Paper 98-3966, AIAA, July 1998.

${ }^{4}$ Sabnis, J. S., "Numerical Simulations of Distributed Combustion in Solid Rocket Motors with Metalized Propellant," J. Propulsion Power, Vol. 19, No. 1, January-February 2003, pp. 48-55.

${ }^{5}$ Dick, W. A. and Heath, M. T., "Whole system simulation of solid propellant rockets," Paper 02-4345, AIAA, July 2002.

${ }^{6}$ Najjar, F. M., Ferry, J., Wasistho, B., and Balachandar, S., "Full-Physics Large-scale multiphase large eddy simulations of flow inside solid rocket motors," Paper 02-4343, AIAA, July 2002.

${ }^{7}$ Rudinger, G., Fundamentals of Gas-Particle Flow, Elsevier Scientific Publishing Company, Amsterdam, 1980 .

${ }^{8}$ Saurel, R., Daniel, E., and Loraud, J. C., "TwoPhase Flows: Second-Order Schemes and Boundary Conditions," AIAA J., Vol. 32, No. 6, June 1994, pp. 1214-1221.

${ }^{9}$ Slater, S. A. and Young, J. B., "The calculation of inertial particle transport in dilute gas-particle flows," Int. J. Multiphase Flow, Vol. 27, 2001, pp. 61-87.

${ }^{10}$ Najjar, F. M., Balachandar, S., Alavilli, P. V. S., and Ferry, J., "Computations of two-pahse flow in aluminized solid propellant rockets," Paper 00-3568, AIAA, July 2000.

${ }^{11}$ Marble, F. E., "Dynamics of Dusty Gases," Ann. Rev. Fluid Mech., Vol. 2, 1970, pp. 397-446.

${ }^{12}$ Groth, C. P. T., Roe, P. L., Gombosi, T. I., and Brown, S. L., "On the Nonstationary Wave Structure of a 35-Moment Closure for Rarefied Gas Dynamics," Paper 95-2312, AIAA, June 1995.

${ }^{13}$ Godunov, S. K., "Finite-Difference Method for $\mathrm{Nu}$ merical Computations of Discontinuous Solutions of the Equations of Fluid Dynamics," Mat. Sb., Vol. 47, No. 3, 1959, pp. 271-290.

${ }^{14}$ Hirsch, C., Numerical Computation of Internal and External Flows, Vol. 2, John Wiley \& Sons, Chichester, 1990.

${ }^{15}$ Barth, T. J., "Recent Developments in High Order K-Exact Reconstruction on Unstructured Meshes," Paper 93-0668, AIAA, January 1993.

${ }^{16}$ Roe, P. L., "Approximate riemann solvers, parameter vectors, and difference schemes," J. Comput. Phys., Vol. 43, 1981, pp. 357-372.

${ }^{17}$ Einfeldt, B., "On Godunov-Type Methods for Gas Dynamics," SIAM J. Numer. Anal., Vol. 25, 1988, pp. 294-318.
${ }^{18}$ Toro, E. F., Spruce, M., and Speares, W., "Restoration of the contact surface in the HLL-Riemann solver," Shock Waves, Vol. 4, 1994, pp. 25-34.

${ }^{19}$ Gottlieb, J. J. and Groth, C. P. T., "Assessment of Riemann Solvers for Unsteady One-Dimensional Inviscid Flows of Perfect Gases," J. Comput. Phys., Vol. 78, 1988, pp. 437-458.

${ }^{20}$ van Leer, B., Tai, C. H., and Powell, K. G., "Design of Optimally-Smoothing Multi-Stage Schemes for the Euler Equations," Paper 89-1933-CP, AIAA, June 1989.

${ }^{21}$ Gottlieb, J. J. and Groth, C. P. T., "Collection of Boundary Conditions for One- and Some MultiDimensional Unsteady Flows of Polytropic Gases," CASI J., Vol. 45, No. 2, June 1999, pp. 161-182.

${ }^{22}$ Groth, C. P. T., DeZeeuw, D. L., Powell, K. G., Gombosi, T. I., and Stout, Q. F., "A Parallel SolutionAdaptive Scheme for Ideal Magnetorhydrodynamics," Paper 95-3273, AIAA, June 1999.

${ }^{23}$ Groth, C. P. T., De Zeeuw, D. L., Gombosi, T. I., and Powell, K. G., "Global Three-Dimensional MHD Simulation of a Space Weather Event: CME Formation, Interplanetary Propagation, and Interaction with the Magnetosphere," J. Geophys. Res., Vol. 105, No. A11, 2000, pp. 25,053-25,078.

${ }^{24}$ Powell, K. G., Roe, P. L., and Quirk, J., "AdaptiveMesh Algorithms for Computational Fluid Dynamics," Algorithmic Trends in Computational Fluid Dynmaics, edited by M. Y. Hussaini, A. Kumar, and M. D. Salas, Springer-Verlag, New York, 1993, pp. 303-337.

${ }^{25}$ Berger, M. J. and Colella, P., "Local Adaptive Mesh Refinement for Shock Hydrodynamics," J. Comput. Phys., Vol. 82, 1989, pp. 67-84.

${ }^{26}$ Greatrix, D. R., A study of combustion and flow behaviour in solid-propellant rocket motors, Ph.D. thesis, University of Toronto, 1987.

${ }^{27}$ Sethian, J. A., Level Set Methods and Fast Marching Methods, Cambridge Monographs on Applied and Computational Mathematics, Cambridge University Press, 2nd ed., 1999.

${ }^{28}$ Vuillot, F., Basset, T., Dupays, J., Daniel, E., and Lupoglazoff, N., "2D Navier-Stokes stability computations for solid rocket motors: rotational, combustion and two-phase flow effects," Paper 97-3326, AIAA, January 1997. 